\title{
Validation of GOME total ozone by means of the Norwegian ozone monitoring network
}

\author{
G. Hansen ${ }^{1}$, A. Dahlback ${ }^{2}$, F. Tonnessen ${ }^{2}$, T. Svenøe ${ }^{1}$ \\ ${ }^{1}$ Norwegian Institute for Air Research (NILU), N-9005 Tromsø, Norway \\ E-mail: georg@nilu.no \\ ${ }^{2}$ University of Oslo, Department of Physics, PO Box 1048, Blindern, N-0316 Oslo
}

Received: 5 June 1998 / Revised: 25 August 1998 / Accepted: 3 September 1998

\begin{abstract}
The Global Ozone Monitoring Experiment (GOME) onboard the ERS-2 satellite has been in operation since July 1995. The Norwegian groundbased total ozone network has played an important role both in the main validation during the commissioning phase and in the validation of upgraded versions of the analysis algorithms of the instrument. The ground-based network consists of various spectrometer types (Dobson, Brewer, UV filter instruments). The validation of the second algorithm version used until January 1998 reveals a very good agreement between GOME and ground-based data at solar zenith angles $<60^{\circ}$ and deviations of GOME total ozone data from groundbased data of up to $\pm 60 \mathrm{DU}(\sim 20 \%)$ at zenith angles $>60^{\circ}$. The deviations strongly depend on the season of the year, being negative in summer and positive in winter/spring, The deviations furthermore show a considerable scattering (up to $\pm 25 \mathrm{DU}$ in monthly average values of $5^{\circ} \mathrm{SZA}$ intervals), even in close spatial and temporal coincidence with ground-based measurements, especially in the high Arctic. The deviations are also dependent on the viewing geometry/ground pixel size with an additional negative offset for the large pixels used in the backswath mode and at solar zenith angles $>85^{\circ}$, compared to forward-swath pixels.
\end{abstract}

Key words. Atmospheric composition and structure (middle atmosphere - composition and chemistry; instruments and techniques).

\section{Introduction}

The ESA Global Ozone Monitoring Experiment (GOME), the first European spaceborne UV-vis spec-

Correspondence to: G. Hansen trometer, was launched onboard the ERS-2 satellite in April 1995. The satellite is in a Sun-synchronous polar orbit with an inclination of $98.5^{\circ}$. Approximate global coverage is reached with GOME within three days, if the instrument is run in the standard mode covering a ground path $960 \mathrm{~km}$ wide. The path is divided into three ground pixels of $320 \mathrm{~km}$ (across orbit) $\times 40 \mathrm{~km}$ (along orbit). The main products of GOME are total ozone and $\mathrm{NO}_{2}$, and the orbit geometry makes the instrument wellsuited for monitoring the scientifically particularly interesting polar ozone layer. A detailed instrument description is given in the GOME Users' Manual (ESA, 1995) and by Callies and Hahne (1998).

GOME takes spectra in the spectral range 237 to $790 \mathrm{~nm}$. To derive total ozone, the differential optical absorption spectroscopy (DOAS) technique is applied. The main philosophy of this method is least-square fitting of an absorption cross section reference spectrum of ozone to the measured spectral radiance in a limited part of the spectrum, yielding a slant column total ozone depending on the geometry of the path of the sunlight. To derive the vertical ozone column density, the slant column has to be corrected for the air mass factor (AMF), which is dependent on various atmospheric parameters, such as temperature and pressure. The exact determination of the AMF is a crucial point (and source of uncertainty) of the algorithm. Temperature does, however, also have an impact via the temperature dependence of the ozone cross section. Furthermore, in the presence of clouds the total ozone has to be corrected for tropospheric ozone below the cloud top which is not seen by GOME. This requires the detection of clouds and the determination of the cloud top altitude.

Total ozone and other products, produced with a still preliminary algorithm (version 1), were validated in a dedicated international campaign in the period July-December 1995. The results of this campaign were presented and discussed in a workshop in January 1996 (ESA, 1996). An interim validation report describing the results of the Norwegian GOME 
main validation project, based on Dobson, Brewer and multi-channel filter instrument data from Oslo, Trom$\mathrm{s} ø$ and $\mathrm{Ny}$-Ålesund, was presented at this workshop and published in the proceedings (Hansen and Dahlback, 1996)

As a consequence of the validation, an improved algorithm (version 2) was implemented in June 1996 and was operated until January 1998. Data produced with this algorithm were validated by a smaller group of validation teams, and partially published since (e.g. Lambert et al., 1997)

In this report, the validation of one year of GOME total ozone data (July 1996 - June 1997) by means of the Norwegian ozone monitoring network is presented. The validation was mainly based on measurements from a new type of instrument, the multi-channel mediumbandwidth UV filter instrument (GUV), calibrated against Dobson measurements at the same site. Instruments of this type are located at Oslo $\left(59.9^{\circ} \mathrm{N}\right)$, Tromsø $\left(69.7^{\circ} \mathrm{N}\right)$ and $\mathrm{Ny}-\AA^{\circ}$ lesund $\left(78.9^{\circ} \mathrm{N}\right)$.

\section{Ground-based measurements}

The Norwegian GOME validation project was initially based on standard ground-based total ozone monitoring techniques, i.e. Dobson and Brewer spectrophotometer measurements. Dobson spectrometer measurements of ozone have a long tradition in Norway. The instrument in Tromsø (e.g. Henriksen et al., 1992) was commissioned in 1939 and has been measuring since, except for a 12-year period from 1972 to 1984. Data from the period 1950 to 1972 have been used to derive pre-CFC ozone reference values, given as monthly means with standard deviations. Two additional Dobson spectrophotometers were set up at Ny-Ålesund, Svalbard, and in Oslo in 1950 and 1978, respectively. In the 1990s, two Brewer instruments were installed in Oslo and Tromsø, increasing the measurement statistics considerably due to the automated operation. For both instruments, generally approved calibration and quality control procedures exist, ensuring their suitability as primary instruments of the Global Ozone Observing System $\left(\mathrm{GO}_{3} \mathrm{OS}\right)$ sponsored by the Word Meteorological Organisation (WMO).

Dobson and Brewer spectrometers do, however, also have disadvantages. The Dobson instrument has to be operated manually, which limits the number of measurements. This disadvantage does not exist for the Brewer spectrometer, but as with the Dobson instrument, it does not yield ozone data of satisfactory quality under unfavourable weather conditions and at low Sun elevation angles, both of which are very common conditions in the Arctic.

Significant progress with respect to these disadvantages was achieved by introducing a new technique, the multichannel moderate-bandwidth filter technique (Dahlback, 1996). Instruments of this type measure global irradiance at several wavelength windows, each $10 \mathrm{~nm}$ wide, in the 300 to $400 \mathrm{~nm}$ spectral range. Using UV radiation transport models, total ozone can be derived with high precision and with a time resolution of a few minutes. Moreover, reliable ozone values can be derived also under unfavourable weather conditions and at solar zenith angles of up to $85^{\circ}$. The quality of the data at zenith angles $>75^{\circ}$ does, however, very much depend on a proper choice of parameters in the radiation transport model, for example on the air mass factor and on the ozone vertical profile. For this reason, all data were checked for systematic changes at large zenith angles. Ozone values showing systematic deviations of more than 5 DU from lower-SZA values were removed.

Furthermore, the GUV data were calibrated against direct-Sun Dobson measurements. Figure 1 shows the ratio between GUV and Dobson direct-Sun measurements for 1996 (upper panel) and the first half of 1997 (lower panel) at Oslo, Tromsø and $\mathrm{Ny}$-Ålesund. It is obvious that the ratio can have both noticeable trends over a year's period as in the case of Tromsø in 1996 or other dependences as for the seasonal one in Oslo in 1996. It is, however, not necessarily the GUV which is the reason for the latter effect; Dobson measurements at
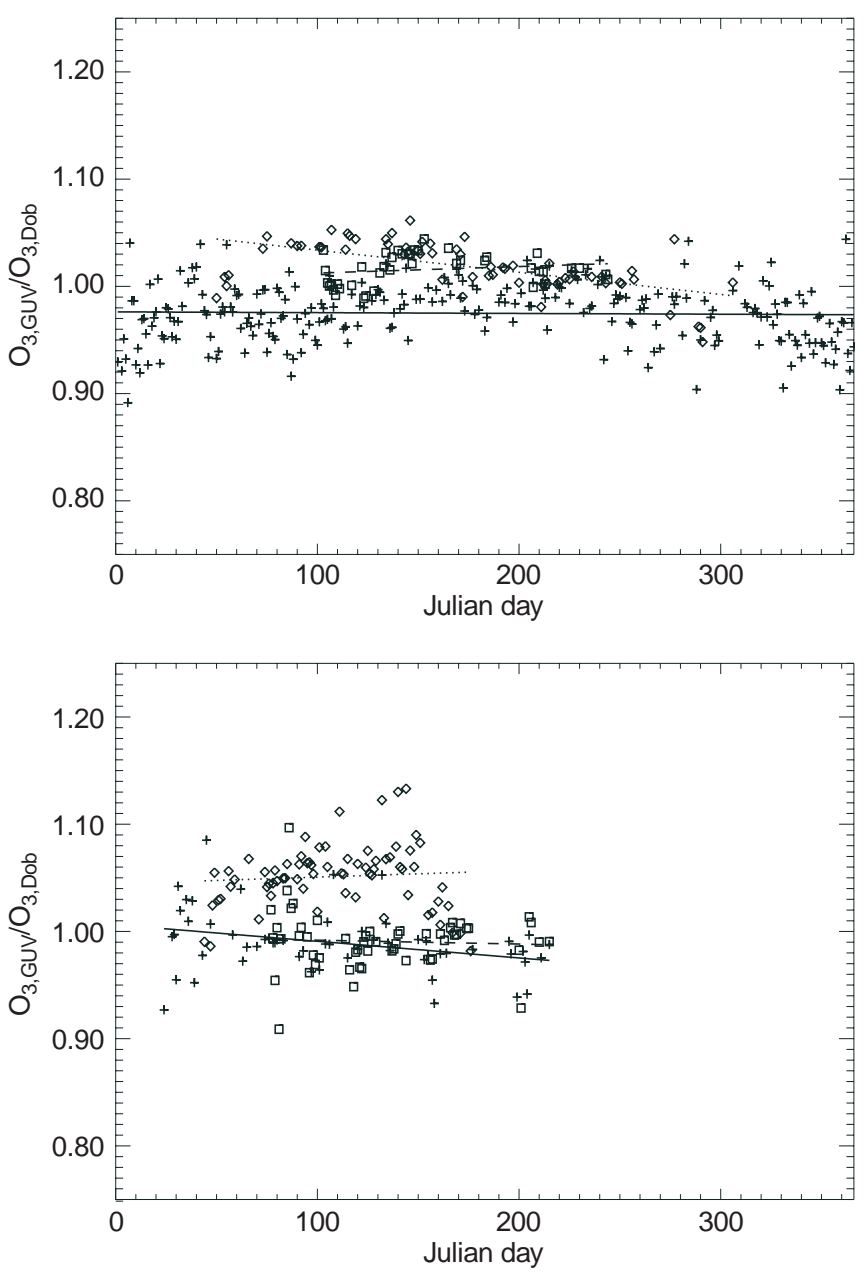

Fig. 1. Ratio between GUV and Dobson daily average total ozone data in 1996 (upper panel) and 1997 (lower panel): Oslo (crosses), Tromsø (diamonds), Ny-Ålesund (squares). Lines: linear regressions for Oslo (solid), Tromsø (dotted) and Ny-Ålesund (dashed) 
solar zenith angles $>80^{\circ}$, which are found in Oslo at winter solstice \pm 1 month, are not very reliable, either. However with a lack of better alternatives, the Dobson measurements were regarded as reference values, also under difficult external conditions, and the GUV measurements were corrected by monthly average correction factors. These vary between 0.95 and 1.06 .

Figure 1 also shows the limitations of ground-based measurements at high latitudes due to the polar night. At $\mathrm{Ny}-\AA$ Alesund at $79^{\circ} \mathrm{N}$, reliable Dobson measurements are possible only from early April to early September. The GUV can extend the usable period by almost one month on either side of the Dobson season.

Figures 2-4 illustrate the availability of data from the instruments used for the validation: while there is usually only one Dobson measurement per day around noon, Brewer and GUV yield by comparison many measurements under good weather conditions, while under less favourable weather conditions the (calibrated) GUV is the only available ground-based data source. At Ny-Ålesund, the GUV is in fact an irreplaceable instrument since there is no Brewer spectrometer. During the summer period it delivers quasi-continuous data records as shown in Fig. 4.

Figure 3 also gives an impression of the differences between measurements by the standard techniques. While long-term agreement to within 1\% between Dobson and Brewer measurements has been reported at middle latitudes and at high and moderate Sun elevation (De Backer and De Muer, 1991), there can be deviations of up to 4\% (10-15 DU) under less favourable conditions (e.g., Dahlback et al., 1997; De Backer and De Muer, 1991). This suggests that after calibration against Dobson measurements, the GUV technique might be equivalent to standard techniques in terms of accuracy.

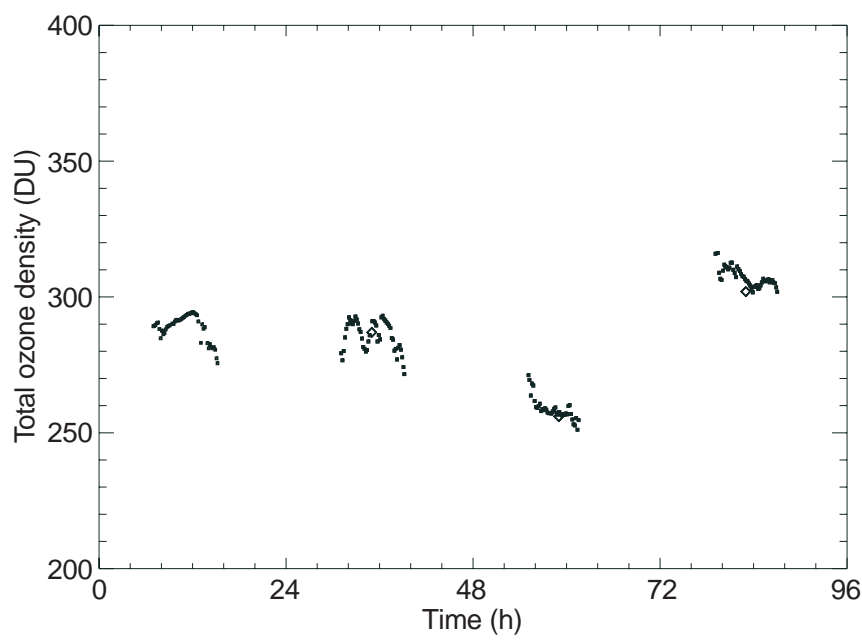

Fig. 2. Ground-based measurements at Oslo in the period October 1-4, 1996. Diamonds: Dobson measurements; dots: GUV measurements

\section{Validation procedure}

Covering the latitude range from 60 to $80^{\circ}$ north, the Norwegian ozone monitoring network is well suited to validate the very critical solar zenith angle (SZA) range of 70 to $90^{\circ}$ of instruments onboard polar-orbiting satellites, such as GOME. The main ground-based data set used for the validation were the GUV data for the period July 1, 1996, to June 30,1997, from Oslo, Tromsø and Ny-Ålesund, calibrated against direct-Sun Dobson measurements at the respective sites.

In the validation procedure, only GOME pixels with a horizontal distance between the pixel centre nadir point and the ground station of less than $300 \mathrm{~km}$ were selected. This value is somewhat arbitrary but was chosen to match the geometry of the GOME ground pixels, the size of which is $320 \times 40 \mathrm{~km}$ in the standard mode (forward swath pixels), while it is $960 \times 40 \mathrm{~km}$ for

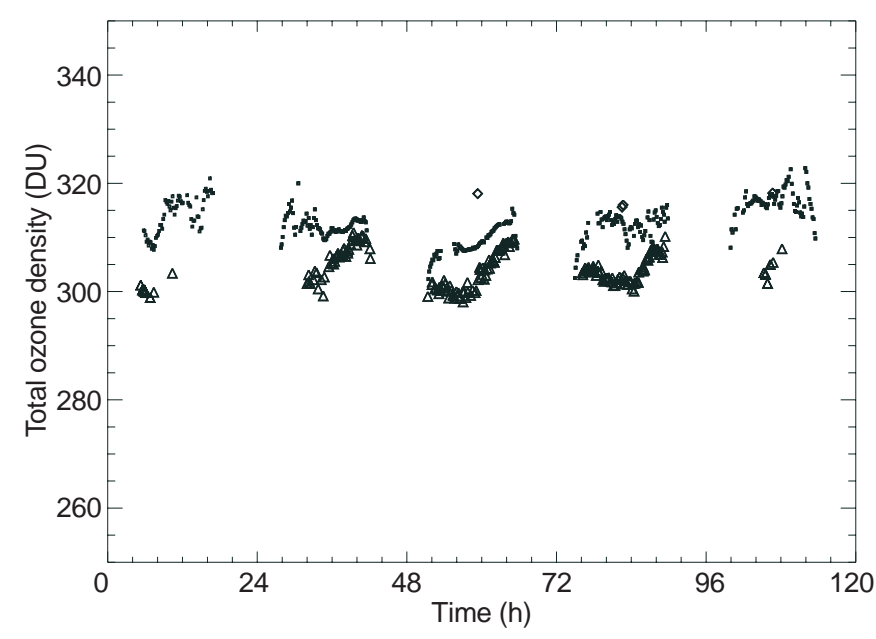

Fig. 3. Ground-based measurements at Tromsø in the period July 27-31, 1996. Diamonds: Dobson measurements; dots: GUV measurements; triangles: Brewer measurements

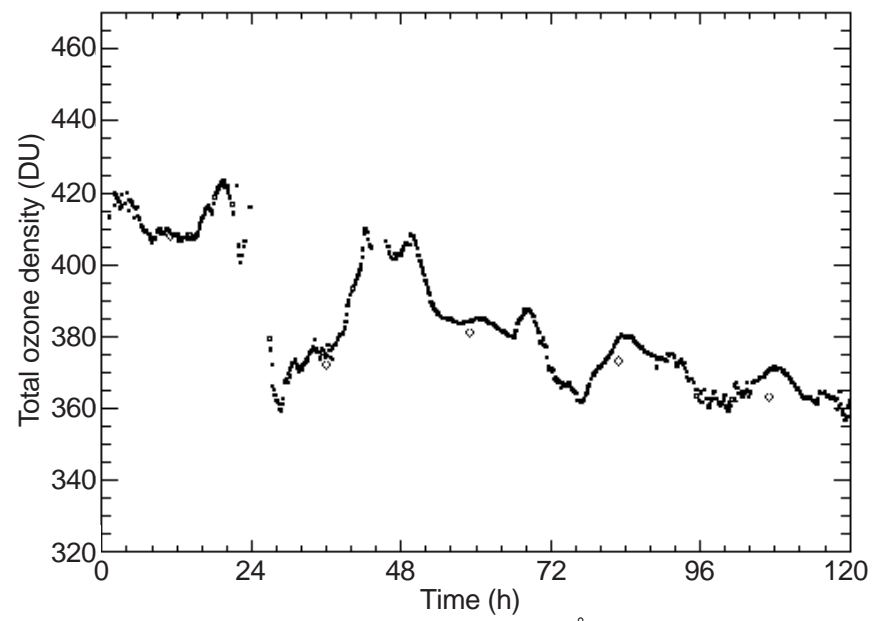

Fig. 4. Ground-based measurements at $\mathrm{Ny}$-Ålesund in the period May 17-21, 1997. Diamonds: Dobson measurements; dots: GUV measurements 
the backward swath pixels and in case of SZA values $>85^{\circ}$. The adequacy of this value was tested using more restrictive distances $(200 \mathrm{~km}, 100 \mathrm{~km})$, yielding only a reduced number of data points but no noticeable change of the result in most cases. Ground-based data were averaged over a time interval of $\pm 3 \mathrm{~h}$ centred around the overpass time of GOME pixels.

\section{Solar zenith angle dependence}

The most important result of the validation, based on several thousand ground-based values and GOME data from about 300 days with up to 6 orbits per day, was a very pronounced dependence of deviations between GOME and ground-based data on SZA at SZA values $>60^{\circ}$. Furthermore, this dependence is strongly dependent on the season of the year. Figure 5 summarizes the SZA dependence, showing monthly average deviations for $5^{\circ}$ SZA intervals of all pixels of $320 \times 40 \mathrm{~km}$ extent or smaller. Crosses denote the months July-December (1996), diamonds the months January-June (1997). The colours for subsequent months are: violet, dark blue, light blue, green, yellow-green and red. Green crosses, for example, denote the deviations in October, while red diamonds denote deviations in June. Due to the geographical location of the Norwegian stations, the sampled SZA range is very limited in the period November-January $\left(>70^{\circ}\right)$.

The deviation data set is fairly consistent in the period July-October. At SZA $<70^{\circ}$ the deviations (GOME total ozone minus ground-based total ozone) agree well within their standard deviations, decreasing slightly from about -10 DU at SZA values of $35^{\circ}$ to about -15 DU at SZA values around $65^{\circ}$. At larger zenith angles, the deviations change systematically from month to month, ranging from almost -50 DU at $85-90^{\circ}$ in July to about $+5 \mathrm{DU}$ in the same SZA range

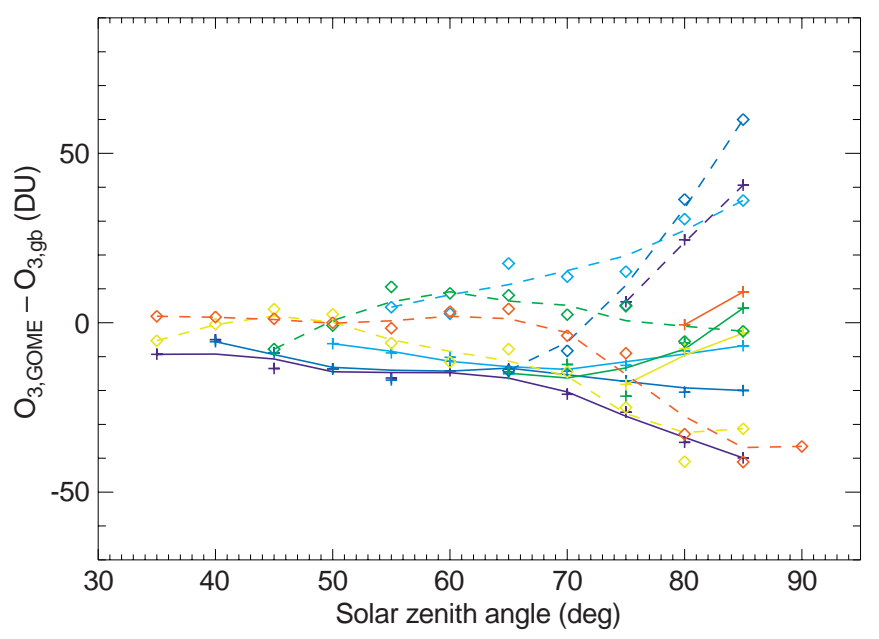

Fig. 5. Deviation of GOME total ozone from GUV values, averaged in 5-degree SZA intervals for all months of the 1-y period investigated. Crosses/solid lines: July-December 1996, diamonds/dashed lines: January-June 1997. Lines: 3-point running mean. Colour code: violet, dark blue, light blue, green, yellow-green, red for subsequent months in October. The deviations continue to rise at the largest SZA values until February/March, reaching maximum positive values of up to +60 DU (about 20\%). From April, the deviations at large SZA again change rapidly, being virtually 0 in April and returning to large negative values in May and June. At solar zenith angles $<70^{\circ}$ the deviations also change from negative values (about -10 DU) in summer/autumn to positive values (about +10 DU) in winter/spring.

On the first GOME validation workshop in January 1996, there was evidence for systematic differences between deviations of forward-swath (typically $320 \times 40 \mathrm{~km})$ and back-swath pixels $(960 \times 40 \mathrm{~km})$ (Koopman and van der Woerd, 1996). For this reason, back-swath pixels were treated separately in this analysis, although the GOME data set used in this analysis is based on another algorithm. Figure 6 shows the total ozone deviations of three selected months for both types of pixels, revealing a noticeable difference. The deviations of large pixels are shifted towards negative values by about 8 DU compared to smaller pixels at SZA values $<75^{\circ}$. This is less than the standard deviation of the differences, but yet it is systematic and found also in other months. At zenith angles $>75^{\circ}$ the difference between large and small pixels becomes insignificant.

The possibility that also the ground-based data set contributes to the deviations is not negligible. A potential source of uncertainty is the GUV data set itself, as there occur systematic deviations at solar zenith angles $>70^{\circ}$ which are eliminated in a somewhat subjective way. For this reason the deviations have been calculated for two months with large deviations of either sign, July and March, using Dobson data. The result is shown in Fig. 7. In the case of July, a temporal offset of the Dobson measurements (usually taken at local noon) from the GOME overpass of up to $6 \mathrm{~h}$ was allowed in order to cover also the large-SZA region. This is

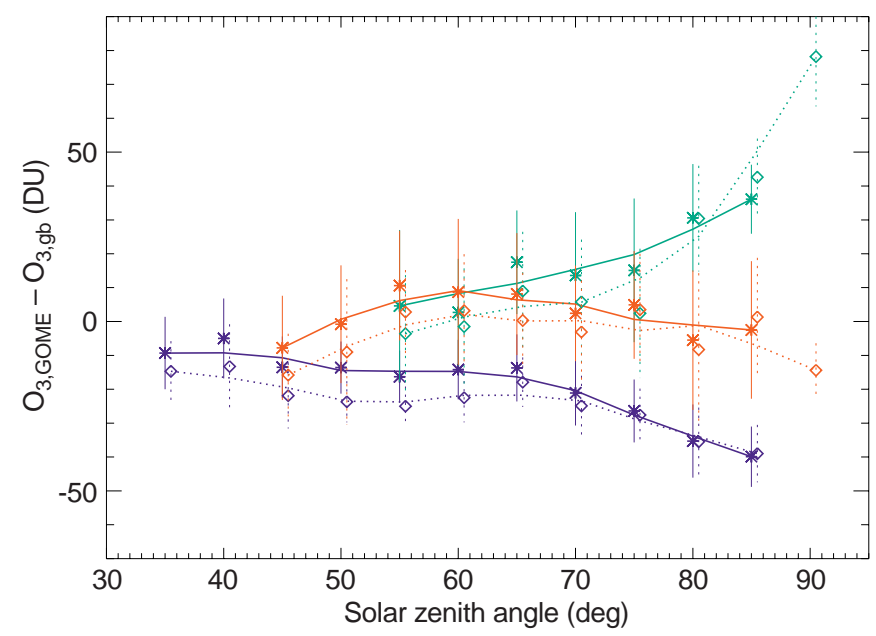

Fig. 6. Deviation of GOME total ozone from GUV values, averaged in 5-degree SZA intervals in July 1996 (blue), March 1997 (green), and April 1997 (red). Solid line/asterisks: forward-swath pixels, diamonds/ dotted line: back-swath pixels. Vertical bars: standard deviations of ozone deviations. SZA values for back-swath pixels are offset by $0.5^{\circ}$ for clarity reasons 


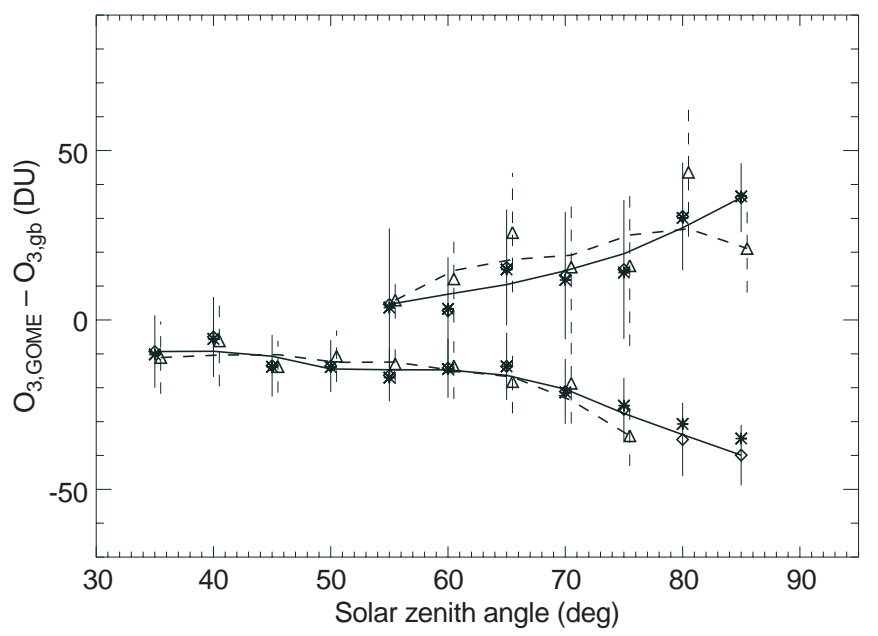

Fig. 7. Deviation of GOME total ozone from ground-based values averaged in 5-degree SZA intervals in July 1996 (lower set of curves) and in March 1997 (upper set of curves). Diamonds (solid line): GUV data averaged over $\pm 3 \mathrm{~h}$ (3-point smoothing); asterisks: GUV data averaged over $\pm 1 \mathrm{~h}$. Triangles (dashed line): Dobson data, in July with an allowed time offset of up to $6 \mathrm{~h}$ (3-point smoothing). Dobson data are offset in SZA by $0.5^{\circ}$ for clarity reasons

acceptable because ozone values are rather stable at this time of the year. The agreement between the GUV- and Dobson-derived deviations is very convincing. In March, the discrepancies between the GUV and Dobson-derived deviations are larger, but also in this case they are well within the standard deviations over the whole SZA range.

Furthermore, it was checked whether the length of the averaging interval of ground-based measurements (relevant for the GUV data) has an impact on the deviations. The result of reducing the interval from $\pm 3 \mathrm{~h}$ to $\pm 1 \mathrm{~h}$ is shown in Fig. 7. The differences between the two data sets are hardly noticeable, except for the two largest SZA intervals in July.

The cause of the deviation and their seasonal variation has been and is further discussed among the scientific teams involved in the validation. A major problem is the climatology used in the GOME algorithm and its implementation therein. The ozone profile climatology might be of particular importance for the seasonally varying SZA dependence of the deviations. Furthermore, in the DOAS approach, an inadequate climatology has a double influence: firstly on the derivation of the slant ozone column directly and secondly on the vertical column via the air mass factor. The temperature is also an important parameter for the ozone cross section. Finally, the GOME algorithm assumes a wave-length-independent UV air mass factor, which is not the case.

\section{Scattering of deviations}

Not only the total ozone deviations between groundbased and GOME measurements vary with season but also the scattering of the deviations. Figure 6 gives three examples of monthly deviations including standard deviation, namely from July, March and April. In July, the standard deviation is in the order of $10 \mathrm{DU}$, which is characteristic for the whole period June-September. In the period February-May, on the other hand, it is almost twice as large, typically 18 DU. There is no significant difference in the standard deviation values between standard-size and large pixels.

The scattering and its seasonal variability can have technical and/or geophysical reasons. The very similar standard deviations of Dobson- and GUV-based analyses shown in Fig. 7 indicate, however, that the groundbased data set is very unlikely to be a source of the scattering. With the Dobson, usually only one value is used as the ground-based reference, in the case of GUV, it is an average of up to 36 single measurements.

Consequently, the scattering occurs in the GOME data set, but not necessarily due to problems in terms of precision of the algorithm. In fact, the most plausible explanation for the seasonal variability of the scattering is the geophysical situation in the region, or more precisely, the insufficient capability of the GOME algorithm to take it into account. In winter/spring, the edge of the polar stratospheric vortex is often located above and north of Scandinavia. The direct effect of this configuration are significant variations in total ozone at rather small horizontal scales. The increased total ozone variability is seen very pronouncedly in long-term total ozone measurements, made, for example, with the Dobson instrument in Tromsø (see. e.g., Fig. 1 in Hansen et al. 1997). Under these circumstances, the choice of the distance criterion as well as the large size of the GOME pixels can have a major impact on the averaged ozone value, compared to the point measurements from ground-based instruments.

The ozone distribution given in Fig. 8 illustrates the distance criterion effect very clearly. On this day (April 2 , 1997), the vortex edge was located about $200 \mathrm{~km}$ south of Tromsø, causing a very sharp horizontal

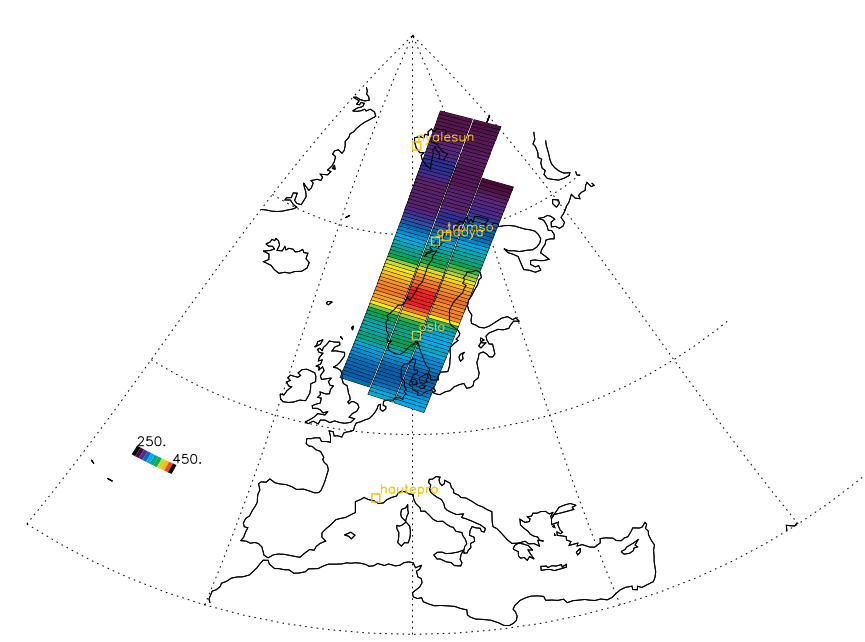

Fig. 8. GOME measurements of total ozone over Scandinavia and Svalbard on April 2, 1997 around local noon. The sharp gradient above north Norway coincides with the edge of the polar stratospheric vortex. Colour scale in Dobson Units 
gradient in total ozone (about $100 \mathrm{DU}$ change over a horizontal distance of about $500 \mathrm{~km}$ ). But also in southern Scandinavia and central Europe there are abrupt changes in total ozone, probably caused by filamentation at the vortex edge or subtropical intrusions. Such events are very common in late winter and spring at middle and high latitudes. Most of the excessive scattering, e.g. in March, can be removed by choosing more restrictive selection criteria for the GOME pixels. If one reduces the maximum allowed distance from 300 to $100 \mathrm{~km}$, the average deviations change very little, while the average scattering of the March data is reduced from $\pm 17.2 \mathrm{DU}$ to $\pm 11.5 \mathrm{DU}$. The July values reveal an average scattering of \pm 9.3 DU. The remaining difference might be an inherent consequence of the pixel size.

The indirect impact of the geophysical situation is that not only total ozone varies considerably under these circumstances. Also other atmospheric parameters (temperature, air density/pressure, ozone vertical distribution) relevant for the GOME total ozone algorithm via the air mass factor correction, change significantly in the vortex edge region. As already mentioned they are not properly represented by the climatology used in the present algorithm, and may also contribute to the large scattering in winter/spring.

At very high latitudes $\left(>75^{\circ} \mathrm{N}\right)$, another source of scattering of deviations was identified after detailed investigation of GOME data. On most days in the period June-August, there are indications of "cold spot" locations, i.e. systematically negatively-deviating pixels. So far, the coast of Svalbard and the northeastern coast of Greenland have been identified. Two examples of this phenomenon are shown in Fig. 9. They both show total ozone values of single pixels in the Svalbard area and at the northeastern coast of Greenland which are between 15 and 20 DU lower than values of adjacent pixels. The pattern of single pixels deviating from surrounding pixels is found in 11 out of 17 orbits on three selected days (July 12, 25, August 4, 1996) with good weather conditions at $\mathrm{Ny}$-Ålesund. Figure 10 shows the GUV measurements of total ozone and "effective" optical thickness on July 25, revealing clear sky (very stable, small optical thickness) and smoothly and slowly declining total ozone values. The total ozone variation over the whole day is smaller than the jumps seen in the GOME data. This makes a geophysical explanation of the phenomenon rather unlikely. It cannot be a simple albedo effect, either, resulting from the (dark) open sea - (bright) snow/ice surface contrast; in this case one would expect to see a signature over the whole of Svalbard and especially at the edge of the pack ice.

It is well-known that in the GOME algorithm version 2.0 the cloud cover (ICFA) is wrong over highly reflecting surfaces because of an incorrect setting in the data processor configuration file. This concerns in particular inland ice, which is found both in Greenland and Svalbard. However, case studies of both total ozone and ICFA have not shown a correlation between the ICFA and the total ozone offsets. This is, for example,
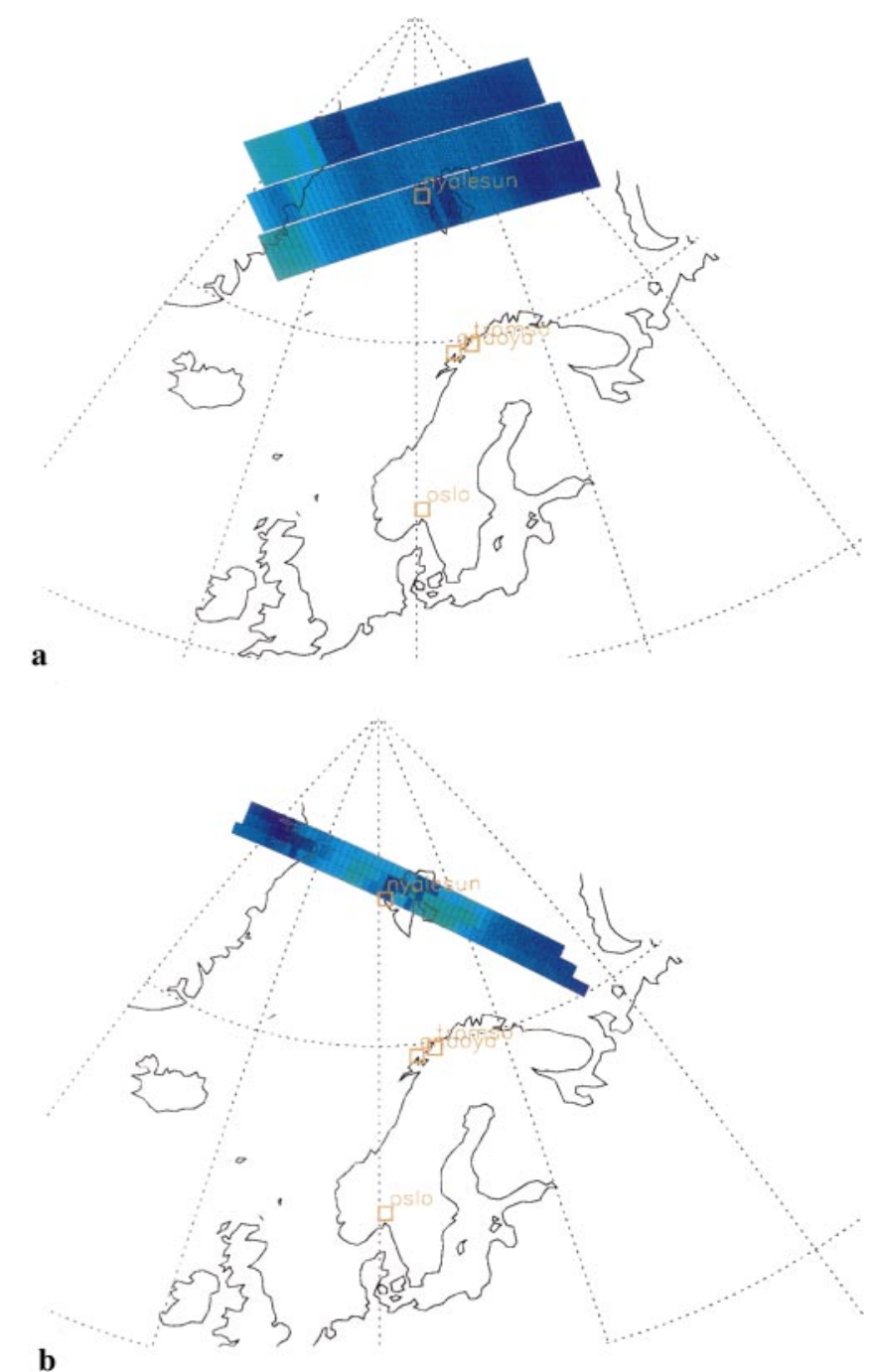

Fig. 9. Selection of GOME pixels around Ny-Ålesund on July 27, 1996, (upper panel) and on June 20, 1997, (lower panel) with pixel sizes of $320 \times 40 \mathrm{~km}$ (upper panel) and $80 \times 40 \mathrm{~km}$ (lower panel). Colour code: as in Fig. 8

indicated in the upper panel of Fig. 9: pixels with negative offset in total ozone are mainly found at the coast, but not all over the inland ice in northern Greenland, while the ICFA is affected all over this region. Since there is no reasonable explanation at present, this phenomenon needs to be investigated in future software upgrading.

\section{Conclusions}

The Global Ozone Monitoring Experiment (GOME) onboard the ERS-2 satellite has during the last three years proven its capability to monitor total ozone with high reliability and, at solar zenith angles $<65^{\circ}$, with an accuracy which is comparable to that of standard ground-based measurements. Validation with the Norwegian ozone monitoring network reveals, however, deviations of up to about $20 \%$, depending on the season 


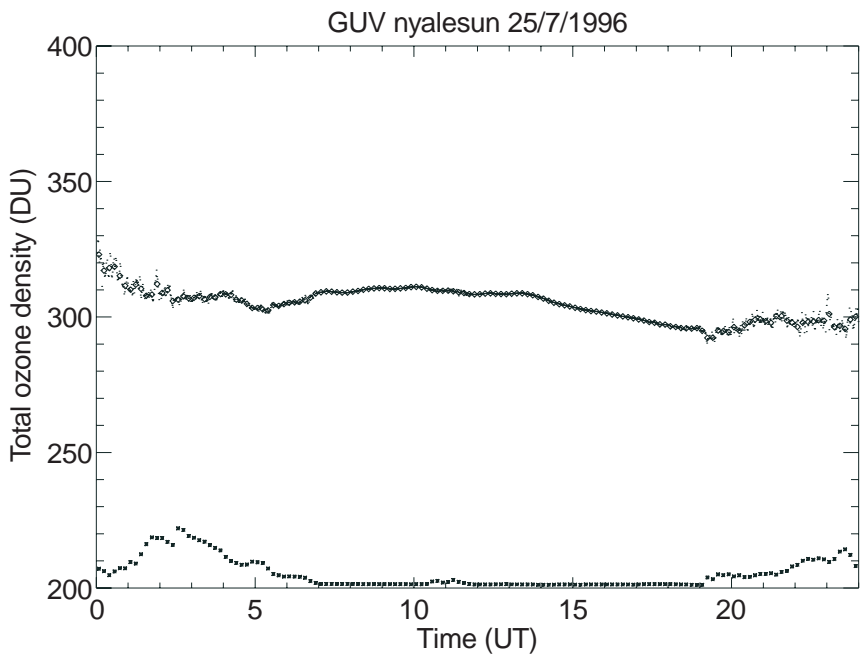

Fig. 10. Total ozone (upper curve) and effective optical thickness + 200 (lower curve) as measured by the GUV at Ny-Alesund on July 25, 1996, (from Hansen, 1998)

of the year, at larger zenith angles, which are common under ozone depletion conditions. The effect is considerably larger than remaining uncertainties in the ground-based data set used in this validation. Both the size and the sign of the deviations found in the study agree well with those studies based on other techniques (e.g., Lambert et al., 1997). Consequently, it must be a problem inherent to GOME measurements.

The most probable source of the deviations between GOME and ground-based data is well-known: it is the climatology and its impacts mainly on the air mass factor and ozone cross section, as well as inherent problems of the AMF technique used in the algorithm version 2.0. Preliminary analysis of the new algorithm version which has been used since beginning of 1998 does not, unfortunately, show a significant improvement. In order to reduce the scattering of the deviations, also special features such as the "cold spots" described have to be investigated in detail. An explanation and removal of these effects will be of great use in order to make GOME and similar instruments on future European satellites, such as ENVISAT, valuable tools in ozone research.

Acknowledgements. The validation software was developed in close cooperation with Rob Koopman, ESA/ESTEC. The GOME data were provided by ESA/ESTEC in cooperation with DLR. We thank all our colleagues from the validation teams, especially J.C. Lambert, IASB, Brussels, Belgium, for very fruitful discussions. The validation project was financially supported by PRODEX funding of ESA/Norwegian Space Centre.

Topical Editor F. Vial thanks J.-C. Lambert and R.M. Koopman for their help in evaluating this paper.

\section{References}

Callies, J., and A. Hahne, GOME - The first European spaceborne UV-vis spectrometer for atmospheric monitoring and research, Proc. 24th Ann. European meeting Atmos. Stud. Opt. Meth., ISBN 82-994583-0-7, 84-89, 1998.

Dahlback, A., Measurement of biological effective UV-doses, total ozone abundance and cloud effects with multi-channel moderate bandwidth filter instruments, Appl. Optics, 35, 6514-6520, 1996.

Dahlback, A., G. O. Braathen, G. Hansen, and T. Svenøe, Surveillance of the ozone layer - Annual report 1996 NILU Report OR29/97, 1997 (in Norwegian).

De Backer, H., and D. De Muer, Intercomparison of total ozone data measured with Dobson and Brewer ozone spectrophotometers at Uccle (Belgium) from January 1984 to March 1991, including zenith sky observations, J. Geophys. Res., 96, 20711 $20719,1991$.

GOME Geophysical Validation Campaign - Final Results Workshop Proceedings, ESA WPP-108, 1996.

GOME Users' Manual, ESA SP-1182, 1995.

Hansen, G., and A. Dahlback, Validation of total ozone measurements with GOME during the main validation phase: the Norwegian project, ESA WPP-108, 199-208, 1996.

Hansen, G., M. Chipperfield, T. Svenøe, A. Dahlback, and U. -P. Hoppe, Evidence of substantial ozone depletion in winter 1995/ 96 over northern Norway, Geophys. Res. Lett., 24, 799-802, 1997.

Hansen, G., Validation of GOME total ozone measurements by means of the Norwegian ozone monitoring network, Proc. 24th Ann. European meeting on Atmos. Stud. Opt. Meth., ISBN 82994583-0-7, 90-95, 1998.

Henriksen, K., T. Svenøe, and S. H. H. Larsen, On the stability of the ozone layer at Tromsø, J. Atmos. Terr. Phys., 54, 11131117, 1992.

Koopman, R. M., and H. J. van der Woerd, Validation of GOME ozone columns using DOBSGOME, and of GOME ICFA cloud cover using ATSR-2, ESA WPP-108, 229-240, 1996.

Lambert. J.-C., M. Van Roozendael, M. De Maziere, P. C. Simon, J.-P. Pommereau, F. Goutail, A. Sarkissian, L. Denis, V. Dorokhov, P. Eriksen, E. Kyrö, J. Leveau, H. K. Roscoe, C. W. Tellefsen, and G. Vaughan, Pole to pole validation of the ERS-2 GOME level 2 products with the SAOZ ground-based network, ESA SP-414, vol. 2, 629-636, 1997. 